\title{
Enrico IV tra Pirandello e Bellocchio
}

\section{Daniela Bini}

La pazzia fu il tema centrale nella vita e nell'opera di Pirandello. Non solo la pazzia della moglie Antonietta, che lo scrittore ebbe modo di osservare e studiare per anni e da cui trasse ampio materiale per i suoi lavori, ma anche, come ha visto bene Gioanola, l'incubo della sua propria latente pazzia, assillarono costantemente Pirandello. ${ }^{1}$ La paura della "bestia" che è in tutti noi e che può scatenarsi da un momento all'altro e sopraffare la ragione: pazzia, quindi, come istinto profondo, forza del rimosso, quello che può spingere "non si sa come" - così c'insegna la commedia omonima - al tradimento e all'omicidio, contro la quale a nulla valgono le briglie della ragione. Ma c'è anche un'altra pazzia, ed è quella che più interessa Pirandello, quella che sembra quasi l'opposto della "bestia" che è in noi, e che finisce paradossalmente per coincidere con essa: la pazzia lucida, quella di cui soffriva anche lui, causata da un uso ossessivo della ragione stimolato da una sensibilità eccessiva. ${ }^{2}$ Pazzo è colui che indaga, che comprende l'assurdità della vita, che vede il nulla dietro le maschere. "Trovarsi davanti a un pazzo", dirà Enrico IV, "significa trovarsi davanti a uno che ti scrolla dalle fondamenta tutto quanto avete costruito in voi, attorno a voi ... la logica di tutte le vostre costruzioni" (Maschere 352). E la pazzia che ci sopraffà in quei "momenti di silenzio interiore, in cui l'anima nostra si spoglia di tutte le finzioni abituali, e gli occhi nostri diventano più acuti e più penetranti". E in quei momenti terrificanti "percepiamo una realtà . . . fuori delle forme dell'umana ragione. Lucidissimamente allora la compagine dell'esistenza quotidiana ... ci appare priva di senso, priva di scopo; e quella realtà diversa ci appare orrida nella sua crudezza impassibile e misteriosa, poiché tutte le nostre fittizie relazioni consuete di sentimenti e d'immagini si sono scisse e disgregate in essa" (L'umorismo 160-61). Davanti al vuoto che si apre di fronte a noi, davanti all'abisso nel quale rischiamo di sprofondare, cerchiamo in uno sforzo supremo di ricostruire l'inganno consueto, di ristabilire le nostre relazioni con le cose che ci circondano, di ricominciare la nostra recita. L'alternativa è la morte o la pazzia.

Come può la società difendersi dalla pazzia di coloro che non solo "percepiscono", come scrive Pirandello nell'Umorismo, ma anche verbalizzano e comunicano al mondo intero l'assurdo dell'esistenza? Con l'ostracismo, l'isolamento, il manicomio; facendogli indossare "il berretto a sonagli" della pazzia. Il marchio, l'etichetta di matto, serve allora ad un duplice scopo; da un lato conferisce calma e sicurezza alla società che si sente protetta nell'attribuire la pazzia ad un'anormalità, nel vederla cioè come una malattia che va esaminata, misurata e magari 
curata con mezzi scientifici; dall'altro il bollo della pazzia conferisce all'individuo la libertà di urlare al mondo le verità più vergognose e dolorose, che la società, che si regge sull'ipocrisia e la finzione, deve per necessità nascondere e negare. Se la "corda pazza", come la chiama Ciampa, indossa "il berretto a sonagli" nella commedia omonima ed è capace ancora di farci sorridere, come già aveva fatto tanti secoli prima la Moría di Erasmo, nell'Enrico $I V$, al contrario, la pazzia indossa la corona regale e ci appare in tutta la sua terribilità. Quando Pirandello scriveva l'Enrico IV nel 1921, aveva già vissuto varie esperienze sconvolgenti. Era arrivato alla decisione penosa di far rinchiudere la moglie in una clinica mentale - dove Antonietta passerà ben quarant'anni, fino alla morte ed è certo possibile e comprensibile che ciò lo sconvolgesse e lo perseguitasse con rimorso. Era vissuto attraverso le atrocità della prima guerra mondiale e la pena causata dalla lontananza del figlio Stefano, partito per il fronte, e infine aveva subito il fiasco a Roma della prima dei Sei personaggi (cominciò a scrivere l'Enrico $I V$ subito dopo quella prima e lo finì in quattro mesi). Tutti questi eventi gli avevano probabilmente fatto toccare con mano l'assurdità delle umane vicende e la sua propria alienazione dal resto del mondo. Pirandello scrive l'Enrico $I V$ guardandosi allo specchio ed esaminando la sua propria pazzia, la sua diversità, la sua follia lucida, la sua esclusione dalla società — anche all'apice del successo infatti Pirandello fu sempre un diverso. ${ }^{3}$

Enrico IV, non a caso unico personaggio del dramma senza un nome vero, vive da vent'anni bloccato in un passato remotissimo, quello del 1071, da quando, in occasione di una mascherata di carnevale, cadde da cavallo e sbatté la testa. Dalla caduta si rialzò convinto d'essere Enrico IV imperatore, di cui indossava la maschera, e in tale convinzione rimase per dodici anni. Il vero dramma inizia al risveglio di Enrico dodici anni dopo, quando, resosi conto del tempo ormai perso per sempre, decide di continuare a vivere nella sua finzione "con la più lucida coscienza" (Maschere 367) - espressione cara a Pirandello - di seguitare ad indossare la maschera, perché l'unica differenza tra Enrico che vive nel suo castello e gli altri che credono di vivere nella vita sta nella consapevolezza del proprio stato da parte del primo e nell'ignoranza dei secondi. Nessuno infatti può evitare di costruirsi non una, ma infinite maschere per vivere; una proliferazione di maschere per le varie occasioni, e non solo quelle che ognuno costruisce di se stesso, ma anche quelle che costruiamo degli altri e nelle quali gli altri non si riconoscono. L'unica differenza tra Enrico e gli altri è che lui ha scelto consapevolmente di vivere la sua finzione, preferendola alla finzione e all'ipocrisia della cosiddetta realtà. Ma come impariamo dagli altri personaggi del dramma, Enrico era già pazzo prima della caduta, pazzo nel senso pirandelliano del termine. La sua è la pazzia di chi non guarda alle cose dal di fuori, ma vi penetra dentro fino al più intimo, con occhi che avevano sconvolto la giovane Matilde; facile all'esaltazione, come dice Belcredi (313), dotato dunque di istinti sensibilissimi e di una lucidissima ragione - connubio infernale e causa di atroci sofferenze. La follia reale, quella causata dalla caduta, quindi, "non fa che portare 
a compimento un destino d'esclusione da sempre iscritto nella sua costituzione esistenziale" (Gioanola 166). ${ }^{4}$

Prima ancora di rivolgersi consapevolmente a Pirandello, Bellocchio attuava con i suoi film un'operazione tipicamente pirandelliana. La tendenza documentaristica del giovane regista - molti sono i suoi film-inchiesta - la preferenza per attori sconosciuti, il rifiuto di "stilemi tipici del film di finzione", servivano in modo più efficace a "sottolineare le tipizzazioni esistenti nella cosiddetta realtà, facendone emergere le finzioni di cui essa è impregnata" (Bernardi 98-99). Se sostituiamo al termine "tipizzazioni" quello di "maschere", ci troviamo in presenza di un tipico discorso pirandelliano. Eे forse proprio il bisogno di mostrare le finzioni della realtà a spingere Bellocchio nel mondo di Pirandello e l'Enrico $I V$ è la scelta più idonea, come accettazione consapevole della propria finzione, superiore (proprio perché voluta e riconosciuta come tale dal soggetto) a quelle in cui la società ci vuole forzare e che ipocritamente chiamiamo realtà.

Bellocchio, regista iconoclasta, impegnato politicamente ma che non si riconosce in nessun partito, feroce fustigatore della società borghese, si sente attratto verso Pirandello non solo da questo aspetto demolitore delle maschere borghesi, ma anche e soprattutto dal tema della pazzia, motivo ricorrente in tutti i suoi film, fin dal primo (Leparulo 69). La pazzia del protagonista de I pugni in tasca è proprio quella di chi scrolla dalle fondamenta le costruzioni borghesi, l'ipocrita finzione della famiglia, ormai solo un involucro privo di contenuto. Pazzia, quindi, come forza rivoluzionaria che per quest'aspetto poteva ancor più affascinare un Bellocchio giovane impegnato politicamente nella lotta della sinistra, che crede nella funzione demistificatrice della rivoluzione. Ma poi? Quale messaggio vuol esprimere il giovane regista con il suo secondo film La Cina è vicina?

Prima di soffermarsi sul film vale la pena di ascoltare quello che lui stesso ci dice su questo passaggio. "I pugni in tasca era stato interpretato piu politicamente di quanto in realtà fosse. Sono stato fissato, definito; e le definizioni sono cose che rischiano di accompagnarti fino alla tomba: 1'arrabbiato, l'iconoclasta, il disgregatore" (Bernardi 4). Anche Bellocchio, quindi, fissato in una forma, in una maschera, proprio come il povero Moscarda in Uno, nessuno e centomila; e allora da bravo umorista, anche lui dà un calcio alla maschera del marxista idealista e ci mostra la grettezza e la meschinità di coloro che di questi ideali si riempiono la bocca. Il film ci presenta un borghese opportunista che si serve del proletariato per ambizione politica, una donna dell'alta borghesia che si lascia ingravidare da un proletario per noia e sfregio della sua classe (e per dispetto al fratello, che come maschio può andare a letto regolarmente con la cameriera, la cui gravidanza, comunque, non dipende da quest'ultimo) e due proletari - figure non certo più nobili dei borghesi - che rinunciano ai loro ideali politici per integrarsi nelle strutture sociali del potere. Le due gravidanze sono chiare metafore di tale operazione, anche se quella della proletaria non è stata opera del ricco borghese, come lei pretende con lui per farsi sposare, ma del proletario (a sottolineare l'impotenza della classe borghese) — una situazione analoga a quella 
che si ritrova nella vicenda di Mattia Pascal. Bellocchio mostra quindi con questo film quanto si era ancora lontani da una seria coscienza rivoluzionaria. Se nel film non c'è un vero portavoce delle sue idee politiche, è perché, come dice il regista echeggiando Montale, ciò che oggi possiamo riconoscere è solo "ciò che non vogliamo" (19). Non c'è dubbio che questa per Bellocchio è una fase che dovrà essere superata. Ma verrà veramente superata? E come? E perché la scelta di Enrico IV tanti anni dopo?

Nel 1974 Bellocchio gira Matti da slegare, un documentario sull'esperienza dell'ospedale psichiatrico di Parma che, sulla scia delle teorie basagliane, aveva tentato l'esperimento di dimettere i malati. L'esperimento fallì presto perché non erano state elaborate le strutture che permettessero ai dimessi l'inserimento nel contesto sociale. Quel che qui interessa, tuttavia, non è tanto l'esperimento sociale, quanto la scelta di Bellocchio di usarlo come materiale per un film. Come dice Bernardi, in Matti da slegare "la follia, paradossalmente, non esiste . . non ha un volto né un'identità fino all'ultima sequenza". Il film ha anche un secondo titolo: Nessuno o tutti, titolo che Bernardi definisce brechtiano, ma che potremmo altrettanto bene definire pirandelliano (94). Anche fuori dai manicomi, i matti continuano ad essere degli alienati e degli esclusi; la cosiddetta liberazione è una pretesa ipocrita in una società che continua a ostracizzare il malato mentale. Ha allora ragione Enrico IV quando sceglie di rimanere chiuso nel suo manicomio regale.

Viene da farsi una domanda: nei dieci anni che passano tra Matti da legare ed Enrico $I V$, Bellocchio ha forse sperimentato la delusione degli ideali sessantottini, il fallimento delle speranze rivoluzionarie? In fondo gli ideali di Bellocchio non sono anch'essi bei concetti, astrazioni costruite dagli uomini per dare un senso alla vita, un tentativo di opporsi e di ribellarsi all'assurdità che ci circonda? Le divise cinesi non sono forse fatte della stessa stoffa della "tonaca santa" a cui il falso "Monsignore" si tiene "fermo aggrappato con tutte e due le mani" mentre intanto senza che ce ne accorgiamo la vita "sguscia" via "come un serpe" (Maschere 325). Comunismo, maoismo, cattolicesimo non sono forse astrazioni, non sono i "castelli ... in aria" di cui parla Cosmo Laurentano ne $I$ vecchi e $i$ giovani? "Soffiate", dice Cosmo, e "ruzzola tutto; perché dentro non c'è niente; il vuoto, tanto più opprimente, quanto più alto e solenne l'edifizio" (Romanzi 55). Ciò nonostante, concluderà Cosmo alla fine del romanzo, come pure Enrico all'apice della sua lucida pazzia, al di fuori di tali costruzioni non c'è nulla, solo il buio spaventoso dell'abisso. La sanità mentale che permette all'uomo di continuare a vivere consiste nel costruire finzioni. "Guai, guai se non vi tenete più forte a ciò che vi par vero oggi, a ciò che vi parrà vero domani, anche se sia l'opposto di ciò che vi pareva vero jeri!" (Maschere 353). Sta nella finzione, dunque, l'unico valore della vita, come già aveva insegnato Leopardi.

Enrico non vive la sua finzione per ingannare gli altri; "la sua finzione non è un inganno, bensì la denuncia degli inganni altrui" (Querci 120). Il suo rifiuto di accettare le regole false e ipocrite delia società in cui vive si è tradotto in rifiuto 
della realtà e della società come tali; di qui l'impossibilità di viverci prima o di tornarci ora. L'accettazione consapevole della maschera impostagli dalla società che l'ha escluso come pazzo, rende tale pazzia simbolo di verità e conoscenza. È questo l'aspetto che Bellocchio sviluppa nella resa cinematografica. La sua preoccupazione politica lo portava naturalmente a sottolineare l'alienazione di Enrico sottolineando la responsabilità della società che l'ha causata e da cui Enrico si autoesclude. Mario Manciotti ha rimarcato, infatti, la volgarità della festa dopo la cavalcata, da cui Enrico si sente e vuole essere escluso, come pure la stupidità farsesca dei cinque visitatori del castello, entrambe invenzioni di Bellocchio, viste in rapporto di causa ed effetto con la pazzia di Enrico. Nella versione filmica, quindi, la società è responsabile della malattia di Enrico. Mettendo l'enfasi su questo aspetto Manciotti tende a prediligere il carattere politico del film e a vederlo come uno sviluppo coerente di un discorso cominciato negli anni '60 (111). Ma Bellocchio fa qualcosa di più del semplice storicizzare la pazzia esistenziale di Pirandello; sviluppa un discorso che non si discosta poi tanto da quello del drammaturgo e che mina alle radici proprio quello stesso messaggio politico che i critici tendono a sopravvalutare.

Se il tema dominante dell'Enrico IV è la pazzia, "il tema dominante di questa pazzia è la rinuncia alla temporalità" (Querci 24). Enrico si è fissato in un frammento di tempo, l'anno 1071, ripete all'infinito la sua breve storia e rifiuta con tutte le sue forze lo scorrere del tempo. Vuole l'eternità attraverso la ripetizione. Il mezzo filmico attua perciò la miglior realizzazione possibile del dramma. Se sulla scena Enrico sarà di volta in volta diverso, perché dipendente dagli attori che lo rappresenteranno, il cinema riuscirà invece a fissarlo per sempre, restituendocelo ogni volta uguale, immutato e immutabile. Se Enrico vive e vuol vivere in un'immagine di sé che permane sempre identica, il mezzo filmico, che traduce in immagini il testo pirandelliano, ne è la forma più idonea. Ma Bellocchio variando il testo fa qualcosa di ancora più pirandelliano. Nel film Enrico non è stato mai pazzo; questo almeno ci lascia intendere il regista, pur non dichiarandolo apertamente. Tale variazione dell'originale mette in evidenza la vera pazzia di Enrico, quella che l'ha sempre contraddistinto, la pazzia esistenziale. Variando il testo di Pirandello, perciò, Bellocchio riesce ad essere ancora più fedele allo spirito di quello.

La rinuncia alla temporalità, l'annullamento del tempo come svolgimento diacronico, è reso dal regista innanzitutto con la mancanza di fedeltà al tempo pirandelliano, fatto questo che sottolinea l'irrilevanza del concetto di tempo in quanto tale. La vicenda è ambientata al giorno d'oggi e il passato è ricostruito con l'aiuto di flashback che vengono giustapposti fin dall'inizio facendo violenza allo svolgimento diacronico del tempo presente reso, in apertura di film, dall'immagine dell'automobile in movimento. L'automobile, simbolo di progresso, di corsa in avanti, sta conducendo i suoi passeggeri verso il passato. La deflagrazione del tempo non potrebbe essere resa in modo più efficace. ${ }^{5} \mathrm{Il}$ film si apre con una elegantissima Mercedes che corre per una strada di campagna. La macchina 
da presa trasporta lo spettatore all'interno della vettura. Per alcuni minuti si sente il suono della radio che trasmette il cambio della valuta, appena percettibile sotto la musica evocatrice della colonna sonora di Astor Piazzolla. Un album di fotografie fatte in occasione della famosa cavalcata e che il dottor Genoni sta studiando è il pretesto all'inizio di una serie di flashback di cui Bellocchio si serve in modo estremamente originale. Fuori dal finestrino, nella campagna circostante, noi spettatori-passeggeri assistiamo alla cavalcata di vent'anni prima. I cavalli galoppano a fianco dell'automobile in una corsa che ci condurrà nel passato di Enrico IV. La giustapposizione di presente e passato non fa che rimarcare di nuovo l'inconsistenza del concetto di tempo.

Il dramma di Pirandello, invece, inizia nel castello di Enrico, con i valletti che danno il background della storia con il pretesto di spiegarla a Bertoldo, il nuovo venuto. L'inconsistenza del tempo viene da Pirandello sottolineata con l'espediente della confusione di Bertoldo che, arrivando, è convinto di trovarsi alla corte di Enrico IV di Francia quattrocento anni dopo. Il dramma teatrale si serve quindi di un lungo preambolo dialogico, mentre nel film è l'immagine a prendere il sopravvento e a parlare in silenzio. Scarno è il dialogo all'interno della macchina come scarna è la capacità comunicativa dei passeggeri. Pur credendo tutti di conoscersi - perfino il dottor Genoni, che pretende di compensare la sua estraneità al gruppo con la preparazione scientifica - i nostri personaggi sono pirandellianamente degli estranei l'uno per l'altro. ${ }^{6}$

Il personaggio con il maggiore spessore psicologico tra $\mathrm{i}$ passeggeri della macchina è senza dubbio Matilde. Le immagini della cavalcata che noi vediamo sullo schermo sono quelle nate dalla sua memoria, stimolata dalla vista delle foto. Solo ora tali immagini sembrano acquistare un senso per lei. Senso che nel momento del vissuto non può essere afferrato e il cui valore viene sottolineato dal personaggio di Frida che, contrariamente al testo, ha nel film un ruolo rilevante. E lei che tenta di cancellare l'errore antico della madre, dando, lei giovane ad un Enrico ormai vecchio, quel "bacin d'amore" che la madre gli aveva allora rifiutato e il cui rifiuto aveva fatto scoccare nel giovane la scintilla della pazzia. ${ }^{7}$ La vita quando la si vive non la si capisce; né il suo senso né il suo valore ci appaiono, anche perché essa in sé non ne ha. Solo a posteriori, nel ricordo, che è creazione, cioè quando la vita non è più, un senso e un valore vengono costruiti dal soggetto che ricorda, cioè falsifica, ricrea; quando il flusso vitale s'è spento e ciò che rimane è solo una maschera vuota. Fermandosi con la cinepresa sulle espressioni del volto di Matilde e giustapponendole alle foto e ai ricordi che le scaturiscono nella mente, Bellocchio riesce, senza parole, a mostrarci quanto maggior senso esse abbiano acquistato ora nel ricordo. I quattro flashback messi in moto dalla memoria di Matilde occupano un posto privilegiato nel film e ciò serve a sottolineare la supremazia del passato in quanto tempo ritrovato, tempo ricreato, tempo significante. Se è vero che la riflessione, l'arte, la forma uccidono la vita che è solo flusso, è anche vero che per conoscerla dobbiamo uscire dalla vita, guardarla dal di fuori e quindi bloccarla in segmenti, proprio come fa la 
macchina cinematografica, e quindi ucciderla (l'epitome di tale procedimento sarà l'operatore Serafino Gubbio). Questa è la tragica dicotomia dell'esistenza umana. Nessuna mediazione è possibile.

I quadri nella sala del trono sono per Enrico il corrispondente delle foto per Matilde: immagini di un passato che è stato ormai fissato per sempre. La differenza tra i primi e le seconde, tuttavia, è che Enrico vive e vuole la sua vita come quelle immagini; ha fissato cioè se stesso e il piccolo mondo attorno a lui in quel tempo. Il quadro è specchio dei suoi desideri. I primi piani di Enrico nella sua immobilità e intensità, sottolineando la somiglianza tra l'Enrico vivo e quello di vent'anni prima rappresentato nel quadro, servono a evidenziare la sua ossessione per il passato, vero protagonista della vicenda. Enrico in esso vive e in esso vuole restare, avendo ormai inconsapevolmente rinunciato alla vita di fuori, quella che scorre, muta e perisce. Questo bisogno di bloccare il tempo e questa ossessione di rimanere chiuso per sempre in una finzione è ben resa da Bellocchio con un'altra modifica, o meglio aggiunta, al testo di Pirandello: la figura del bambino spesso giustapposta ad Enrico e con la quale egli ovviamente si identifica. Un'aggiunta al testo, abbiamo detto, ma un'aggiunta quasi scaturita dal testo stesso. Il dottor Genoni, infatti, così descrive la malattia del pazzo, in questo caso di Enrico: "si può essere sicuri che un pazzo . . . può notare un travestimento davanti a lui; e assumerlo come tale; e sissignori, tuttavia crederci; proprio come fanno i bambini, per cui è insieme giuoco e realtà. Ho detto perciò puerile. Ma è poi complicatissimo in questo senso, ecco: che egli ha, deve avere perfettamente coscienza di essere per sé, davanti a se stesso, una immagine: quella sua immagine là!" (Maschere 334). Ma in fondo che altro siamo se non immagini? Che altro se non creazioni? Quelle che ci costruiamo noi e quelle che di noi si fanno gli altri. L'immagine che Enrico ha creato di sé è altrettanto reale per lui, quanto quelle che di lui si fanno gli altri, perché è lui che vuole crederci. Questo è quel che ci ha insegnato Laudisi in Cosi è se vi pare, che la realtà non consiste nei documenti, nei fatti, ma nell'animo, nei sentimenti, nei desideri di ogni individuo, o, con le parole di Nietzsche, solo nelle interpretazioni. "Il fantasma che noi ci creiamo", dice Laudisi, "ha la stessa consistenza della realtà [. . . . E non potrà essere distrutta, questa realtà [. . .] da nessun documento, poiché [noi tutti] ci respir[iamo] dentro, la ved[iamo], la sent[iamo], la tocc[hiamo]" ( $M a$ schere 1041). La famosa scena di Laudisi allo specchio è la manifestazione del procedimento di autocoscienza che lo porta all'affermazione che l'immagine che lui vede nello specchio non corrisponde a quelle che di lui vedono gli altri, ma che non sono per questo meno vere, come anche Moscarda dolorosamente scoprirà. Enrico ha abolito gli specchi, ma ha messo al loro posto il ritratto di se stesso giovane, nel costume di Enrico IV, vicino a quello di Matilde da lui amata. La vicinanza desiderata acquista realtà nel mondo dell'immaginario di Enrico. Significativamente Bellocchio, invece, lascia ad Enrico uno specchio, dove egli si guarda, mostrandoci quanto sia ancora più consapevole e voluta la sua finzione. 
L'immagine del bambino è dunque un'invenzione di Bellocchio, suggerita, come abbiamo visto, dal testo di Pirandello. Enrico con questo si identifica in un gioco che vorrebbe eterno. Bimbo anche lui, ha fissato, immortalato la famosa cavalcata in un bellissimo cavallo di legno, sul quale spesso monta, come su quello di una giostra, che ripete all'infinito lo stesso tragitto, tornando sempre al punto di partenza. Se la vita intensa, irrazionale e violenta trova una facile simbologia nel cavallo - la cavalcata rappresenta la corsa sensuale e impetuosa di un gruppo di giovani verso la vita - , il cavallo di legno di Bellocchio è simbolo della vita fissata, morta. Chi non sa o non vuole vivere la vita reale, lo fa vicariamente, nella finzione della mascherata; il carnevale, come giustificazione del proibito, luogo privilegiato per lo sfogo del represso. Le immagini di Enrico sul cavallo finto si prestano, perciò, ad una lettura umoristica. Se da un lato richiamano subito alla mente quadri e statue di condottieri famosi, simbolo di potere e di permanenza, dall'altro esse rappresentano l'eterna umana "pupazzata" fissata in una giostra puerile.

Per sottolineare il contrasto tra lo scorrere del tempo e la stasi dell'attimo fissato, Bellocchio inserisce nel film un altro elemento nuovo di elevata tensione poetica, che apre una nuova dimensione di significato: la fantastica stanza degli uccelli di balza bianca - in sintonia con quella altrettanto fiabesca della lana cardata, anch'essa bianca - con lo sfondo luminosissimo di una finestra aperta; uccelli dalle ampie ali, quasi vele mosse dal vento sul mare dell'immaginario di Enrico e dello spettatore. Uccelli, quindi, simbolo di vita per eccellenza, ma finti; vita fissata e, in quanto tale, uccisa; ulteriore personificazione dell'essenza ossimorica della realtà. Oltre il vento è anche Enrico a muovere questi uccelli, qui metafora dell'artista che crea davanti agli occhi del pubblico la sua finzione. Il testo di Pirandello è in fondo anche questo: una metafora della creazione artistica. Il tempo fissato non ha valore in quanto passato, storia, ma in quanto creazione. ${ }^{8}$

Ma il dramma di Pirandello si può anche leggere nel modo opposto, e cioè ponendo l'enfasi sull'altro polo della dicotomia, e dire che è la vita che alla fine entra a sconvolgere la finzione di Enrico; la vita con i suoi istinti e le sue passioni. E la vista di Frida che provoca il gesto omicida, ed è forse la paura della vita a rinchiudere di nuovo Enrico nella sua finzione, come afferma Gioanola, la sua incapacità di vivere (167). Ma non potremmo anche leggere il gesto omicida di Enrico contro Belcredi come metafora dell'arte che uccide la vita? Se Enrico rappresenta l'artista che vive nella sua finzione, Belcredi rappresenta la vita nella sua foga e sensualità, alter-ego di Enrico, "doppio maligno", come giustamente lo chiama Gioanola ("Mito" 137). Ed è probabile che proprio questa sia l'intenzione dell'artista, l'offerta al suo pubblico di un finale ambiguo, contraddittorio, umoristico. Enrico è l'erma bifronte che da una faccia ride del pianto dell'altra. E insieme l'artista che deliberatamente sceglie di restare fissato nella sua finzione, e nello stesso tempo l'inetto, colui che ha paura ed è incapace di vivere. Le due interpretazioni finiscono per coincidere. La vita, aveva detto Pirandello, o si vive o si scrive. Lo scrittore e l'inetto sono la stessa persona. E Bellocchio 
sembra porre l'enfasi sull'Enrico artista se sceglie di sdrammatizzare il finale, eliminando l'omicidio di Belcredi e presentandoci un Enrico costantemente in controllo di sé e della situazione. Non c'è colpo di scena nel finale; ed è Enrico che sceglie di restare nella sua finzione senza esserne forzato da fattori esterni.

Bellocchio è ben consapevole del suo pirandellismo con cui ha sempre dovuto scendere a patti. La scelta di portare sullo schermo proprio l'Enrico $I V$ non è certo stata casuale, ma deve forse essere letta come una confessione, come una dichiarazione di autocritica. Così il regista parlava qualche anno prima in un'intervista: "L'esasperato individualismo dei miei film mi crea degli scrupoli. Di conseguenza sento l'esigenza di riacchiappare il reale che sta veramente fuori di me . . di tirarlo dentro, nel discorso personale, per una specie di sfiducia nella forza di quest'ultimo" (Bernardi 8). È possibile che qualche anno dopo la sfiducia si sia spostata proprio su quel reale che Bellocchio aveva precedentemente tentato di "tirar . . . dentro, nel discorso personale" e che questo gli si sia rivelato solo una grande "pupazzata". Forse anche Bellocchio, in fondo, ha preferito alla mascherata ipocrita del reale quella consapevole della finzione artistica. ${ }^{9}$

University of Texas at Austin

\section{NOTE}

1 Il libro di Gioanola, Pirandello e la follia, è senza dubbio lo studio più completo sul tema.

2 In questa interpretazione che corregge in parte Gioanola, seguo i recenti studi di L.A. Sass, che nel suo Madness and Modernism propone una lettura della schizofrenia come provocata non da una deficienza o confusione mentale, come si è a lungo ritenuto, bensì da "hyperreflexivity" e "acute selfconsciousness" (8). Ciò che viene a mancare in tali condizioni patologiche è la capacità di agire. La paralisi che ne consegue è una paralisi dell'azione fisica, non di quella mentale, ed essa è causata proprio da una iperattività della riflessione.

3 Maurice Valency ha commentato che nell'Enrico IV Pirandello "distilled . . a all the bitterness with which a lifetime of misfortune had inspired him, and all the scom and indignation of an unforgiving nature" (224).

4 Il critico mette l'enfasi sull' incapacità di vivere di Enrico, che io credo sia soltanto suggerita da Pirandello, non asserita con certezza. Enrico, infatti, rifiuta la vita non solo perchê è incapace di vivere, ma anche perché vuole la permanenza, il senso, la forma, cioè rifiuta la vita in quanto flusso e decadimento.

5 Bermardi ha sottolineato il ruolo centrale che ha l'automobile, "grande strumento di emancipazione", nei film di Bellocchio, cominciando già da I pugni in tasca (35). In Enrico $I V$, invece, l'idea di progresso rappresentata dalla macchina è ovviamente messa in discussione dall'uso ironico che ne vien fatto.

6 Per i tratti umoristici del personaggio del dottor Genoni nel dramma di Pirandello, si rimanda all'ottimo saggio di Douglas Biow.

7 L'osservazione sul bacio come elemento catalizzatore è della mia amica e collega Millicent Marcus. Varie idee in questo saggio sono il prodotto delle fruttuose discussioni avute con lei, di cui desidero ringraziarla.

8 Se Biow ha ragione nell' affermare che Pirandello demistifica la storia e mostra nell'Enrico $I V$ che la storia è malattia (167-70), il discorso va sviluppato con la considerazione che tale 
demistificazione è quella di una storia come documento obiettivo, testimone di eventi. Pirandello non crede alla storia, come dice il dottor Fileno nei "Colloqui con i personaggi", ma alle storie, quelle create dagli uomini, in cui ognuno mette la sua anima e il sangue, come l'Ignota di Come tu mi vuoi, che vuole darsi un passato, creare di sé una storia. Enrico riscatta la sua storia in quanto la vive come finzione. Come in Cosi è se vi pare, anche qui si parla di documenti perduti; Enrico attribuisce la distruzione dei documenti della sua vita all'opera dei suoi nemici, ed è per questo che ora si appresta a dettame di nuovi al fedele fraticello Giovanni, anche lui ben consapevole della finzione. La storia che Enrico detterà allo scriba Giovanni sarà la sua versione degli avvenimenti dell'epoca, sarà la sua finzione.

9 Con il film Enrico $N$ Bellocchio realizza qualcos'altro che Pirandello avrebbe probabilmente apprezzato; l'annullamento della parola attraverso la fusione di immagini e musica. Nell"“Azione parlata", scritta nel 1929 dopo i primi rozzi tentativi di cinema parlato, Pirandello vede nel cinema un potenziale infinito nella realizzazione musicale; un cinema, come ha scritto Fink, "che si libera della soggezione alla narrativa e al dramma . . . per immergersi tutto nella musica". Con le parole di Pirandello: "Ecco: pura musica e pura visione. I due sensi estetici per eccellenza, l'occhio e l'udito, uniti in un godimento unico; gli occhi che vedono, l'orecchio che ascolta, e il cuore che sente tutta la bellezza e la varietà dei sentimenti ... sommuovendo il subcosciente che è in tutti . . . . Cinemelografia, ecco il nome della vera rivoluzione: il linguaggio visibile della musica" (Fink 279). (Anche la citazione di Pirandello è nel saggio citato sopra [280].) Pirandello parlava qui del cinema a servizio della musica, di un'operazione cioè simile a quella realizzata da Walt Disney con il film Fantasia; ciò nonostante avrebbe apprezzato anche l'operazione contraria, la resa musicale di stati d'animo che non possono essere adeguatamente espressi con le parole, ma devono essere solo suggeriti o evocati. Nelle scene iniziali del film, la parola è ridotta al minimo, e le confuse frasi di background che vengono dalla radio ne sottolineano l'essenza paradossale, in quanto assolutamente fuori fase con i pensieri e gli stati d'animo dei passeggeri della macchina. Le stupende immagini della campagna e dei giovani a cavallo nei ricchi costumi d'epoca sono accompagnate dall'efficace musica di Astor Piazzolla. Le espressioni del volto di Matilde sono tradotte in musica, non in parole, ma potremmo anche dire, se non sapessimo che la creazione di Pirandello precede la creazione di Piazzolla, che è la qualità dei toni di questa stessa musica ad ispirare i sentimenti di Matilde e quindi a creare una storia. Alla musica ricorrerà Enrico in vari momenti della vicenda per calmarsi e sognare. Degno discepolo di Nietzsche, Pirandello sa benissimo che la parola per sua natura falsifica. Un'arte di suoni ed immagini è, forse, ciò che meglio può avvicinarsi alla resa fedele del mondo sentimentale e istintuale dell'individuo.

\section{OPERE CITATE}

Bellocchio, Marco. China is near. Trad. Julian Green. New York: Onion Press, 1969.

Bernardi, Sandro. Marco Bellocchio. Firenze: La Nuova Italia, 1978.

Biow, Douglas. "Psychoanalysis, History, Marginality: A Study of Violence and Disease in Pirandello's Henry $I V$ ". Italica 66 (Summer 1989): 158-75.

Fink, Guido. "Voce di macchina e voce del padre ('Se il film parlante abolirà il teatro')". Pirandello saggista. Palermo: Palumbo, 1982. 272-82.

Gioanola, Elio. "Mito e follia nell'Enrico $I V$ ". La persona nell'opera di Pirandello. Atti del XXIII Convegno Internazionale. Milano: Mursia, 1990. 121-40.

. Pirandello e la follia. Genova: il melangolo, 1983.

Leparulo, William E. "From Theater to Film: Bellocchio's and Pirandello's Enrico IV". Canadiar. Journal of Italian Studies 13 (1990): 69-74. 
Manciotti, Mario. "Enrico IV: la rinuncia alla metafora". Bianco e nero 45 (luglio-settembre 1984): 107-11.

Pirandello, Luigi. Maschere nude. Vol. 1. Milano: Mondadori, 1958-86. . Tutti i romanzi. Vol. 2. Milano: Mondadori, 1973.

. L' umorismo. Milano: Mondadori, 1986.

Querci, Giovanna. Pirandello: l' inconsistenza dell' oggettività. Bari: Laterza, 1992.

Sass, Louis A. Madness and Modernism. New York: Basic Books, 1992.

Valency, Maurice. "On Henry IV". A Companion to Pirandello Studies. Ed. John Di Gaetani. New York: Greenwood Press, 1991. 223-29. 